\title{
Retos terminológicos y traductológicos en el atentado contra Charlie Hebdo: el lenguaje eufemístico (inglés-español)
}

\author{
Alba Montes Sánchez \\ Universidad de Córdoba \\ 132mosaa@uco.es
}

https://dx.doi.org.10.12795/futhark.2020.i15.10

\begin{abstract}
Fecha de recepción: 15.03 .2020
Fecha de aceptación: 30.05 .2020

Resumen: Gobiernos y organizaciones internacionales contemplan el terrorismo como un problema de repercusión mundial, un factor complejo que se ha abordado desde numerosos ámbitos de estudio. En cierto modo, el tipo de terrorismo perpetrado en la actualidad logra sus objetivos gracias, en gran parte, a la información facilitada a través de los medios de comunicación, pues otorgan cobertura inmediata sobre sus actuaciones a nivel planetario, lo que da a entender que la relación entre el terrorismo y los medios de comunicación está cada vez más reforzada, puesto que, si cierta información no resulta publicada, los actos terroristas parecer no ser noticia. La presente investigación plantea un acercamiento a la terminología del campo del terrorismo a través de los eufemismos. Para ello, en primer lugar, este estudio presenta la relación existente entre los medios de comunicación y el terrorismo desde un enfoque lingüístico. En este sentido, esta investigación parte de un corpus comparable (inglés-español) constituido por textos periodísticos sobre el atentado terrorista contra Charlie Hebdo. Con ello, se pretende analizar y resolver las cuestiones lingüísticas que se originan en inglés y español desde una perspectiva traductológica, con el objetivo fundamental de evidenciar el volumen de eufemismos que emplea la prensa española e inglesa en este campo de especialidad. Finalmente, se observa cómo los resultados arrojados por el análisis podrían redundar en beneficio de los profesionales que trabajan en la investigación o en la transferencia de información sobre contenidos terroristas, como periodistas, lingüistas y traductores e intérpretes.
\end{abstract}

Palabras clave: Eufemismos, terrorismo, traducción, medios de comunicación, terminología. 


\title{
Terminological and traductological Challenges in the Attack against Charlie Hebdo: Euphemistic Language (English- Spanish)
}

\begin{abstract}
Governments and international organizations consider terrorism as a problem of global repercussion, a complex factor that has been approached from numerous fields of study. In a certain way, the type of terrorism perpetrated today achieves its objectives thanks, in large part, to the information provided through the media, as they provide immediate coverage of their actions throughout the world. This fact implies that the relationship between terrorism and the media is increasingly reinforced, since, if certain information is not published, the terrorist acts appear not to be news. This research addresses an approach to the terminology of the field of terrorism through euphemisms. To do this, first, it is presented the relationship between the media and terrorism from a linguistic approach. In this sense, our study starts from a comparable corpus (EnglishSpanish) integrated by journalistic texts about the terrorist attack against Charlie Hebdo. This is intended to analyze and resolve the linguistic issues emerged in English and Spanish from a translation perspective, with the fundamental aim of showing the volume of euphemisms used by the Spanish and English press in this field of specialty. Finally, it is observed how the results of the analysis could be beneficial for the professionals who work in the investigation or in the transfer of information on terrorist contents, such as journalists, linguists and translators and interpreters.
\end{abstract}

Keywords: Euphemisms, terrorism, translation, media - terminology.

Sumario: I. Introducción: La traducción y el terrorismo en un mundo globalizado. 2. El tratamiento del terrorismo en los medios de comunicación: los eufemismos. 2.I. El lenguaje eufemístico en terrorismo. 3. Compilación del corpus y metodología. 4. Análisis contrastivo inglés-español. 5. Consideraciones finales.

\section{Introducción: La traducción y el terrorismo en un mundo globalizado}

Los estudios de Pegenaute (20/2) centran su investigación en el establecimiento del vínculo o relación entre los profesionales de la traducción y la interpretación y el terrorismo. Dicho autor sitúa como punto de partida la globalización (2012: 398), que ha contribuido con numerosos avances positivos en lo referente a cuestiones sociales, políticas, tecnológicas y económicas. No obstante, desde una perspectiva muy poco optimista, Pegenaute expone que la globalización ha brindado "consecuencias colaterales negativas", puesto que ha favorecido la perdida de la identidad cultural local o, visto de otra forma, la 
creación de una identidad más internacional), así como el desarrollo del terrorismo internacional:

\begin{abstract}
La globalización ha sido causa y efecto de dos elementos determinantes y, en apariencia, contradictorios: por una parte, la instauración del inglés como lingua franca; por otra, la necesidad cada vez más acuciante de salvar las barreras impuestas por la diversidad lingüística mediante la traducción y la interpretación. No valerse de estas herramientas para acceder a discursos que de otra forma resultarían opacos puede tener consecuencias calamitosas, incluso para la seguridad nacional [...] (Pegenaute, 2012: 398-399).
\end{abstract}

Según argumenta Pegenaute (2012: 405), cuando la globalización y los conflictos bélicos rebasan las fronteras lingüísticas, culturales y nacionales, "resulta sintomático el desarrollo cada vez más frecuente de estudios sobre sociología de la traducción y la atención a los aspectos éticos de su actividad". Esto se remonta a los atentados terroristas del II de septiembre de 200I en Nueva York, los cuales marcaron un antes y un después en la figura del traductor-intérprete. El gobierno estadounidense vislumbró la relevancia de la traducción en las investigaciones llevadas a cabo por los servicios de inteligencia y la importancia del intérprete en juicios e interrogatorios. Nuestra profesión ha ido adquiriendo relevancia no solo en lo referente a las investigaciones, sino también a través de los medios de comunicación al favorecer la cobertura mediática. Esto se ha ido evidenciando a lo largo del presente siglo, pudiéndose apreciar el trabajo del intérprete-traductor en el II-M, en los atentados de París aquella noche del I 3 de noviembre de 2015 o en el atentado de La Rambla de Barcelona el 17 de agosto de 2017, entre otros. Esto ha resaltado la labor de los traductores e intérpretes en la resolución de conflictos.

En palabras de Shariffan (2007), destacamos como dato relevante la responsabilidad ideológica y sociopolítica que, supone el tratamiento de determinados vocablos en contextos en los que la carga ideológica está muy presente, como sucede en ejemplos como "yihad", "compromiso", "concesión" o "Eje del mal". Estas palabras, al ser tratadas de forma errónea en el proceso de traducción o interpretación, podrían ocasionar graves consecuencias políticas o sociales, pues están cargadas de elementos culturales. A los profesionales de nuestro gremio les ocurre algo similar a los periodistas, quienes, según Veres (2006), la transmisión de la información conlleva una gran responsabilidad y compromiso social, en este contexto incluso más que en otros. Asimismo, el traductor se puede plantear una serie de interrogantes éticos sobre la información que debe transmitir, pues el traslado de información proveniente de grupos y organizaciones terroristas de forma completamente objetiva podría desatar graves consecuencias, como mínimo sociales. el lingüista como el informador con su principal herramienta de trabajo: el lenguaje. ya que. Lo mismo ocurre con la tarea. Por consiguiente, el lenguaje es la principal herramienta de periodistas y traductores, por lo que se debe tener conciencia, según señala Casado (I99|: 56),

$\begin{array}{ll}\text { Futhark I5 (2020) } & \text { I45 }\end{array}$


de que "las palabras no determinan nuestra forma de pensar, pero de alguna forma nos predisponen a favor de ciertas líneas de pensamiento".

\section{El tratamiento del terrorismo en los medios de comunicación: los eufemismos}

Pese a la multitud de perspectivas de estudio desde las que se ha estudiado el fenómeno del terrorismo (político, sociológico, psicológico, académico, jurídico, etc.), en el enfoque lingüístico son escasas las investigaciones planteadas. Igualmente ocurre con los medios de comunicación, que es la vía de difusión más directa con la sociedad (véase Gadarian, 2010), o destacando el carácter simbiótico, como argumenta Zurutuza (2006: 76), de la relación entre el terrorismo y el periodismo. Según plantea Veres (2006), es un aspecto primordial destacar la adherencia y atracción que los terroristas sienten hacia los medios de comunicación. En este sentido, Eco (1978) afirma que "el terrorismo es un fenómeno de nuestra época, de la época de los medios de comunicación de masas”. Además, insinúa que, sin la existencia de estos medios de comunicación masivos, determinados actos no se producirían puesto que su principal objetivo es llegar a ser noticia, hacerse eco a través de los medios de comunicación. En este punto nos vemos obligados a cuestionar, de nuevo, la delimitación del concepto "terrorismo" en su relación con los mass media o medios de comunicación, pues como indica Rodrigo (I991: 27), "el terrorismo es rehén de los medios de comunicación y estos lo son de él".

En cierto modo, el tipo de terrorismo - en concreto el yihadismo - que nos azota en la actualidad logra sus objetivos gracias, en gran parte, a la información facilitada a través de los medios de comunicación, pues otorgan cobertura inmediata sobre sus actuaciones a nivel planetario, lo que da a entender, en palabras de Veres (2006: 290) que es bastante evidente la relación existente entre el terrorismo y los medios. Al parecer, hasta que un atentado que ha sido perpetrado o cierta información emitida por los grupos u organizaciones terroristas no se publica, no son noticia, ya que no se ofrece difusión a escala mundial.

\section{I. El lenguaje eufemístico en terrorismo}

En el presente subapartado nos gustaría destacar, en primer lugar, que el fenómeno terrorista presenta numerosos problemas de tipo lingüístico en relación con los medios de comunicación. En este contexto, los grupos y organizaciones terroristas recurren, de forma muy habitual, al empleo de eufemismos para trasladar la información. El término eufemismo proviene del latín euphemismus y este del griego euphemismós (eu, bien, y phemí, decir), lo que según Gallud (2005: 12I) se asocia de forma automática a vocablos como 'eufonía' o 'eufuismo', los cuales nos transmiten una noción de belleza. Según este autor, un eufemismo es una "estrategia discursiva que consiste en substituir una expresión dura, vulgar o 
grosera, por otra suave, elegante o decorosa". Por su parte, la Real Academia Española lo define como "una manifestación suave o decorosa de ideas cuya recta y franca expresión sería dura o malsonante". Es decir, su uso se produce en casos en los que la expresión real puede resultar desagradable, con el fin de aminorar la negatividad que su significado de por sí conlleva. A continuación, presentamos una relación de términos con sus eufemismos correspondientes dentro de la temática del terrorismo.

\begin{tabular}{|ll|}
\hline TÉRMINOS & EUFEMISMOS \\
\hline Alto el fuego & Tregua \\
\hline Asesinato, crimen o muerte & Ejecución \\
\hline Atentado & Acción armada, operación o ataque \\
\hline Bomba & Artefacto explosivo \\
\hline Bombardeo & Salida \\
\hline Comando terrorista & Célula terrorista \\
\hline Delincuentes & Radicales \\
\hline Destrozos & Desperfectos \\
\hline Extorsión & Impuesto revolucionario \\
\hline Fugitivo o prófugo & Refugiado \\
\hline Genocidio & Limpieza étnica \\
\hline Matar, asesinar & Liquidar, neutralizar \\
\hline Secuestrado o rehén & Prisionero \\
\hline Terrorismo & Lucha armada \\
\hline Terrorista & Soldado, activista \\
\hline Víctimas civiles & Daños colaterales \\
\hline
\end{tabular}

Tabla I. Términos y eufemismos en terrorismo (Fuente: Elaboración propia).

Según Veres (2006: 292), la emisión de una noticia en cualquier soporte supone un acto semejante a cualquier otra acción humana, pero en el caso de tratarse de un suceso de contenido terrorista, el riesgo aumenta respecto a otras noticias informativas, por lo que el tratamiento de este léxico debe emplearse con cautela. A menudo profesionales de la esfera política y periodística hacen uso de los eufemismos en temas que, según Kalounerová (2015), son considerados tabúes culturales, como por ejemplo para hacer referencia al origen étnico, cuestiones bélicas o políticas o las drogas. En cuanto al terrorismo se refiere, es común observar cómo los profesionales de la información sustituyen las T-words o "palabras-T" por un lenguaje decoroso en el que reinan los eufemismos. 
Los medios de comunicación y su manera de informar sobre el terrorismo han contribuido de forma muy significativa a paralizar y ofuscar todavía más la eterna política de definir al autor de los actos de violencia como terrorista o como combatiente por la libertad (Hoffmann, 1990: 4I).

En este aspecto, Veres (2006: 296) argumenta que tanto los terroristas como, en ocasiones, los propios periodistas que de forma inconsciente recurren este tipo de palabras, "además de alejar del ámbito los aspectos ajenos a los intereses del terror, consiguen un objetivo todavía más ambicioso [...] la legitimación de su empresa".

\section{Compilación del corpus y metodología}

En primer lugar, a fin de contrastar el empleo de eufemismos en contextos periodísticos redactados en español e inglés, hemos decidido limitar la compilación de textos a un determinado atentado. Consideramos que de esta forma el análisis podrá concluir unos resultados más minuciosos y detallados. Por consiguiente, hemos consultado varios periódicos ingleses y españoles que han publicado información relativa al atentado perpetrado contra Charlie Hebdo, un semanario satírico francés de ideología de izquierdas fundado el 1992. Este atentado fue perpetrado por los hermanos yihadistas Chérif y Saïd Kouachi, quienes irrumpieron armados en la sede de París de Charlie Hebdo el 7 de enero de 2015 y mataron a 12 personas, II personas que trabajaban en la redacción y un policía en una calle lindante.

El corpus comparable (inglés-español) se compone de una compilación de noticias relativas a dicho atentado desde el 7 hasta el 31 de enero 2015 con el propósito de obtener una muestra equilibrada. La prensa consultada es de ámbito nacional, siendo así periódicos digitales que cuentan con un gran impacto mediático en ambos países y creando un amplio abanico de ideologías políticas hacia las que pueden estar orientados. Para compilar el corpus en inglés, hemos recogido textos de los diferentes periódicos: BBC News, Evening Standard, The Guardian, The Mirror y The Telegraph. En cuanto a la prensa española, destacamos los siguientes: Economía Digital, El Mundo, La Vanguardia, Okdiario y Público. En suma, la recolección de datos nos ha permitido compilar un total de 25 textos en inglés y 27 en español, con 31 y 33 casos de eufemismo léxico respectivamente.

La metodología seguida para el análisis de los términos eufemísticos es similar a la seguida por Crespo (2016) y consta de los siguientes pasos:

A. Inicialmente hemos seleccionado la información en ambas lenguas que son objeto de estudio, para lo que se ha recurrido a la búsqueda avanzada en los citados periódicos, dentro de la fecha estipulada. 
B. Seguidamente, con el fin de clasificar los datos que eran de interés para el presente estudio, establecimos unas categorías en las que diferenciar los términos que considerábamos eufemismos dentro del campo del terrorismo. Según Kalounerová (2015), los eufemismos se emplean principalmente para referirse al término 'terrorista' (attacker, warrior, rebel, etc.), terrorismo (armed conflict, massacre, man-made disaster, etc.) o al conjunto total de la organización terrorista (Boko Haram fighters, The Palestinian militant goup, Hamas men, etc.). No obstante, también pueden observarse para crear un lenguaje decoroso que se refiera a las acciones militares, como por ejemplo los métodos de interrogación (stress position, coercive interrogation, sleep management, etc.), para hacer referencia a las víctimas (collateral damage, massacre, soft target, neutralised, etc.) o para otros vocablos que tienen relación directa con el terrorismo (raid, bloodshed, savagery, etc.). Por tanto, en aras de acotar nuestra investigación, hemos compilado textos atendiendo a eufemismos que pueden ser empleados para sustituir al vocablo terrorist.

C. Por último, hemos procedido a presentar una serie de deducciones basadas en datos objetivos pero que contarán con generalizaciones y cierta subjetividad. Nuestro objetivo es dar cuenta del verdadero significado de los eufemismos observados y cómo se emplean en el contexto del terrorismo dentro del discurso periodístico. Por tanto, pese a ser un corpus representativo, las conclusiones obtenidas de este análisis cualitativo carecen de fuerza a nivel cuantitativo.

\section{Análisis contrastivo inglés-español}

Según Kalounerová (2015), los términos 'terrorista' y 'terrorismo' a menudo se emplean de forma incorrecta por los medios de comunicación, ya que existe cierto prejuicio contra estos vocablos y se pretende evadirlos. Esta inclinación hacia los eufemismos o el doublespeak o lenguaje ambiguo pretende suavizar la información que se transmite; no obstante, existen grandes y pequeños matices entre los diferentes vocablos que son utilizados como sinónimos. De hecho, según Rodrigo Alsina (1991: 134), existen cuatro tipos de discurso sobre el terrorismo cada uno de los cuales hace referencia a los actores o terroristas y sus actos de forma diferente: político (terroristas, bandas fascistas, acción revolucionaria o atentado terrorista); militar (grupo operativo militar, comando armado, acción armada o acciones de guerra); jurídico (secuestradores, asesinos, acción criminal o muestra de gangsterismo) y patológico (banda de locos, grupo degenerado, hecho irracional o salvajada).

Consecuentemente, en aras de limitar nuestro estudio, nos centramos en el análisis del vocablo "terrorista" (en inglés, terrorist), para el cual según Kalounerová 
(2015) se han encontrado al menos 20 substituciones que los medios de comunicación emplean como eufemismos: activists, assailants, attackers, bombers, captors, commandos, criminals, extremists, fighters, group, guerillas, gunmen, hostagetakers, insurgents, kidnappers, militants, perpetrators, radicals, rebels, separatists.

(1) Charlie Hebdo attackers: born, raised and radicalised in Paris. ${ }^{1}$

(2) Son dos hermanos, de 32 y 34 años, pertenecientes a la región de París y un tercer atacante con apenas 18 años. ${ }^{2}$

Attacker se define como "a person who uses violence to hurt someone", en español, “persona que emplea la violencia para dañar a alguien”. El origen del verbo attack se remonta al año 1660 y proviene del francés attaquer, que a su vez tiene como origen el florentino italiano attacare (battaglia). Para este análisis, consideramos relevante, en primer lugar, establecer la diferenciación entre ataque y atentado. Ataque es un término genérico que aparece frecuentemente en textos sobre esta materia debido, principalmente, al calco que realizamos en el proceso de traducción del inglés al español del término attack. La Fundéu recuerda que en español ya disponemos de una palabra para referirnos a este tipo de actos, por ello, aunque el empleo de la expresión "ataque" o "ataque terrorista" no es incorrecto, es preferible recurrir a la construcción "atentado terrorista" en la lengua meta. De igual modo, al referirnos a la persona o personas que perpetran el atentado, existen numerosas opciones de traducción. No obstante, en español y, más en concreto en la prensa española, el término attacker suele tener como equivalente el sustantivo "atacante".

(3) The French media reports that the assailants fled from the scene in a black car and are now on the run. ${ }^{3}$

(4) El fiscal de París afirma que los asaltantes del ataque contra 'Charlie Hebdo' se identificaron como miembros de $\mathrm{Al}$ Qaeda. ${ }^{4}$

El vocablo assailant tiene sus orígenes en el año 1530 del francés, el cual se emplea como participio de presente del verbo assalir. Según el Collins Dictionary,

\footnotetext{
I Disponible en: https://www.theguardian.com/world/20I5/jan/I2/-sp-charlie-hebdo-attackers-kidsfrance-radicalised-paris.

${ }^{2}$ Disponible en: https://www.publico.es/internacional/identificados-autores-del-ataque-revista.html.

${ }^{3}$ Disponible en: https://www.mirror.co.uk/news/world-news/charlie-hebdo-paris-terror-attack-4934389.

${ }^{4}$ Disponible en: https://www.lavanguardia.com/internacional/20150107/5442242/294/testigo-charliehebdo-al-qaeda.html.
} 
este término se define como "one who assails or attacks; attacker", lo que se traduce al español como "aquella persona que asalta o ataca; atacante".

(5) These extremists must know that they can never silence freedom of speech, for it is a stronger weapon than any they'd dare to carry. ${ }^{5}$

(6) Dos días después del ataque contra la revista, otro extremista se atrincheró con rehenes en un supermercado kosher. ${ }^{6}$

El vocablo extremist tiene su origen en 1840 al adherir dicho sustantivo el sufijo -ist. Su significado es "one who goes to extremes, a supporter of extreme doctrines", en español, "aquel que va a los extremos, que defiende las doctrinas extremistas". El extremismo es definido por la RAE como "la tendencia a adoptar ideas o actitudes extremas, especialmente en política". El término que analizamos aparece con asiduidad en los textos que tratan sobre materia terrorista; no obstante, no tienen por qué estar directamente relacionados con el concepto de terrorismo, ya que el extremismo no siempre conlleva la perpetración de actos terroristas. Por tanto, hay que destacar en este contexto la diferencia entre "actos extremistas" o "actos radicalizados" y "actos terroristas".

(7) Hostage-takers: gunmen Said Kouachi (left) and Cherif Kouachi (right). ${ }^{7}$

(8) Cuatro rehenes y el secuestrador mueren en el asalto de la policía a la tienda judía. ${ }^{8}$

Hostage, que significa en español rehén, tiene sus orígenes a finales del siglo XIII y proviene del francés ostage "persona dada como seguridad o rehén" (ôtage en francés moderno); o bien, del latín tardío obsidanus "condición de ser retenido como seguridad", de obsesos "rehenes". El sentido político o terrorista que posee en la actualidad fue otorgado en el año 1970. En inglés, hostage-taker es definido como "a person held by one party in a conflict as security that specified terms will be met by the opposing party.", es decir, "una persona retenida por una de las partes en un conflicto como garantía de que la otra parte contraria cumplirá los términos especificados". Según Veres (2012: 4), la gran parte de los atentados, secuestros y asesinatos protagonizados por organizaciones terroristas parten de "la

\footnotetext{
${ }^{5}$ Disponible en: https://www.bbc.com/news/live/world-europe-307/0777.

${ }^{6}$ Disponible en: https://okdiario.com/internacional/la-revista-charlie-hebdo-lanzara-una-edicion-especialpor-el-aniversario-del-atentado-terrorista-que-sufrieron-en-el-2015-48265.

${ }^{7}$ Disponible en: https://www.bbc.co.uk/news/av/world-europe-30754340/charlie-hebdo-hunt-kouachibrothers-killed-in-assault.

${ }^{8}$ Disponible en: https://www.elmundo.es/internacional/20I5/0I/09/54afcad52260IdIfl58b4584.html.
}

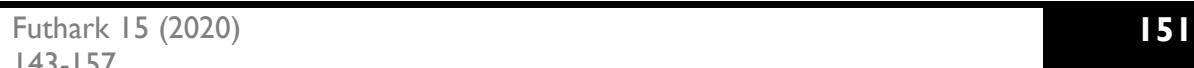


creación de significación, de cierto efecto eco y en una resemantización del sentido". Entendemos que en el contexto que analizamos, los autores del secuestro tienen como fin privar la libertad de los secuestrados. De hecho, Rodrigo Alsina (I99I: 129) identifica tres tipos de actos con finalidad terrorista: a) actos contra la vida (aquellos que tienen como resultado la muerte), b) actos contra la libertad (como serían los secuestros), y c) contra la propiedad (contra bienes inmuebles con el fin de causar daños materiales).

(9) Factory owner held in Paris printworks siege reveals he made captors coffee and tended to their wounds ${ }^{9}$

(10) El captor ha exigido la liberación y el fin del cerco contra los hermanos Kouachi, presuntos autores de la matanza en Charlie Hebdo, como condición para no matar a los rehenes ${ }^{10}$.

El origen del vocablo inglés captor se remonta al latín captor del año 1680 , cuyo sustantivo agente es captus, participio pasado del verbo latín capere, que significa "coger o tomar". Segun el Collins Dictionary, un captor es "a person who captures (someone or something) or holds captive", es decir, "una persona que arresta (algo o a alguien) o lo mantiene cautivo". En español, "captor" no es un vocablo al que se recurra fácilmente, especialmente en prensa, ya que existen otros sustantivos más estandarizados como secuestrador, cautivador o incluso raptor que actúan como sinónimos. No obstante, también se puede observar el empleo de este eufemismo en textos periodísticos en español, quizás como consecuencia de la influencia anglosajona en este contexto específico.

(11) Ten journalists and two policemen killed after the attack by three gunmen on the Charlie Hebdo magazine office in Paris. ${ }^{11}$

(12) Dos hombres armados han entrado este miércoles por la mañana en la redacción del semanario francés Charlie Hebdo y han disparado contra las personas que estaban en ese momento en la oficina, situada en París. ${ }^{12}$

\footnotetext{
${ }^{9}$ Disponible en: https://www.standard.co.uk/news/world/factory-owner-held-in-paris-printworks-siegereveals-he-made-captors-coffee-and-tended-to-their-996994l.html.

${ }^{10}$ Disponible en: https://www.lavanguardia.com/internacional/20150109/54423164262/hombre-armadotoma-rehenes-tienda-kosher-paris.html.

" Disponible en: https://www.telegraph.co.uk/news/worldnews/europe/france/II331902/Charlie-Hebdoattack-Frances-worst-terrorist-attack-in-a-generation-leaves-12-dead.html.

12 Disponible en: https:/www.economiadigital.es/politica-y-sociedad/doce-muertos-en-el-asalto-a-un-
} semanario-frances-satirico-con-el-islam_16439|_102.html. 
El vocablo inglés gunman está compuesto por dos sustantivos: gun, que se traduce como arma, y man, que significa hombre. Su origen se remonta a los comienzos del inglés americano, en concreto el empleado por los indígenas guerreros ${ }^{13}$. Según el Collins Dictionary, "a gunman is a man who uses a gun to commit a crime such as murder or robbery ${ }^{14,}$, es decir, "una persona armada es aquella que emplea un arma para cometer un delito como un asesinato o un robo". A la hora de emplear un término cuyo significado sea similar en español, hemos observado que no existe un único vocablo que actué como equivalente, si no que se recurre a la construcción de sustantivo+adjetivo en lugar de sustantivo+sustantivo, como ocurre en inglés, que sí podría actuar como equivalente en este contexto especializado. En español, hemos compilado varios textos periodísticos que emplean la combinación "hombre armado" o "persona armada", ofreciendo así una descripción de quién perpetra el atentado.

(13) The perpetrators of the Charlie Hebdo massacre in Paris should be not be described as "terrorists" by the BBC [...]. ${ }^{15}$

(14) " $\mathrm{Si}$ los perpetradores son musulmanes, podría tener repercusiones negativas en los musulmanes de Europa entera y en Francia en particular. Los extremistas de derechas intentarán utilizar este incidente para conseguir sus fines políticos". ${ }^{16}$

El vocablo perpetrator tiene sus orígenes en el año 1560 y proviene del latín perpetrator, definido como "a person who perpetrates, or commits, an illegal, criminal, or evil act" 17 , es decir, "una persona que perpetra o comete un acto illegal, criminal o malvado". Para traducir este término, recurrimos al Código Penal español. En el Art. 83 está tipificada la "coautoría" y se dicta lo siguiente "Cuando varias personas concurran a la ejecución de un hecho punible, cada uno de los perpetradores (autores) y de los cooperadores inmediatos queda sujeto a la pena correspondiente al hecho perpetrado". Por tanto, los sustantivos "perpetrador" y "autor" actúan como sinónimos en contextos penales y, por consiguiente, podemos observar el empleo de uno u otro como eufemismo para evitar así la denominada "palabra-T".

${ }^{13}$ Disponible en: https://www.etymonline.com/word/gunman.

${ }^{14}$ Disponible en: https://www.collinsdictionary.com/es/diccionario/ingles/gunman.

${ }^{15}$ Disponible en: https://www.telegraph.co.uk/news/bbc/l I369/04/We-must-not-call-Charlie-Hebdokillers-terrorists-says-BBC-boss.html.

${ }^{16}$ Disponible en: https://www.lavanguardia.com/internacional/201501 10/54423195892/hermanosmusulmanes-condena-ataque-charlie-hebdo.html.

17 Disponible en: https://www.dictionary.com/browse/perpetrator. 
Según Martínez (200I) y orientado al ejemplo (14), cuya cita corresponde a un activista político, el eufemismo puede tener cuatro raíces: la pusilanimidad, la cursilería o pedantería, los neologismos y tecnicismos y el interés político-social. En este caso concreto, consideramos que el cuarto aspecto es el que se refleja en el presente ejemplo. El eufemismo, que de acuerdo con dicho autor no es más que un reflejo del politically correct estadounidense, se emplea en el lenguaje político-social $y$, como consecuencia, por tanto, en el texto periodístico.

(15) At his trial in 2008 he was described a reluctant holy warrior with his lawyer saying he was sacred of being killed in Iraq $[\ldots] .{ }^{18}$

(16) Los terroristas se han convertido en guerreros, hiperarmados y mataron deliberadamente a cuantas personas había en la redacción. ${ }^{19}$

El sustantivo warrior halla su origen en el año 1300, proviene así del antiguo guerrero del norte de Francia (guerroieor) "guerrero, soldado, combatiente, aquel que hace la guerra". Este vocablo inglés se define como "a fighter or soldier, especially one in former times who was very brave and experienced in fighting", es decir "un guerrero o soldado, especialmente alguien que con anterioridad ha sido muy valiente y experimentado en la lucha". El empleo de este término, tanto en español como en inglés, supone abordar con anterioridad ciertas cuestiones. En primer lugar, según afirma Wilkinson (1974: 17), el hecho de que determinados actos terroristas puedan confundirse con otro tipo de delitos ordinarios, como por ejemplo el asesinato, los daños y el crimen de guerra, hace destacar que la finalidad del terrorismo no es propagar miedos sociales extensos, sino únicamente méritos puntuales. Por ello, siguiendo las guías de Álvarez (2019), un buen comienzo para delimitar la frontera existente entre la definición del término terrorismo sería marcar las diferencias entre guerrilleros, criminales $y$ asesinos con aquellos denominados "terroristas" o autodenominados "guerreros":

[...] los guerrilleros son grupos numéricamente más grandes, que actúan como unidad militar, que atacan fuerzas militares enemigas, que capturan territorios, los sostienen y buscan establecer una forma de soberanía o de control geográfico y poblacional (los terroristas no funcionan abiertamente como unidades armadas, no intentan capturar ni sostener territorios); los criminales emplean la violencia para obtener dinero y bienes materiales, con fines egoístas y personales, no les preocupa la opinión pública (el terrorista no actúa por

${ }^{18}$ Disponible en: https://www.standard.co.uk/news/world/charlie-hebdo-massacre-suspects-have-stolena-car-and-are-on-the-run-again-9966876.html.

${ }^{19}$ Disponible en: https://www.lavanguardia.com/internacional/20150108/54423120744/no-es-buenmomento-para-ser-musulman-en-francia-tahar-ben-jelloun.html. 
motivos personales e incluso su violencia parece perseguir fines altruistas, y busca entregar un mensaje con sus acciones); los asesinos tienen un objetivo intrínsecamente idiosincrásico, egocéntrico y personal (el terrorista tiene fines políticos, es altruista y un intelectual violento).

A esto se suma el adjetivo que acompaña al vocablo: holy. Según Flori (2004: 7- 10) la conocida Holy War o "Guerra santa" es un conflicto que tiene su origen en las diferencias y discrepancias religiosas, en concreto entre aquellos extremistas religiosos que defienden la existencia de un solo Dios, así como la de aquellos lugares que consideran sagrados. De forma popular, se presentan como ejemplos de guerra santa las cruzadas y la yihad islámica. En consecuencia, para tratar el término warrior debemos atender al adjetivo que le acompaña tanto en español como en inglés, como consecuencia de la influencia léxico de las propias organizaciones terroristas con ejemplos como el (I5) que hace referencia a la "guerra santa".

\section{Consideraciones finales}

A la hora de hacer un balance final consideramos imprescindible hacer referencia a la procedencia de los textos utilizados. Como hemos mencionado anteriormente, la fuente de nuestro material lingüístico son periódicos ingleses y españoles que publicaron información relativa al atentado de Charlie Hebdo sucedido en la capital francesa en enero de 2015. Estamos ante el análisis de un léxico contextualizado en actos terroristas del que hacen uso los medios de comunicación, por tanto, estudiamos y analizamos el uso del léxico eufemístico en el lenguaje periodístico centrado en un campo de estudio en concreto. Por consiguiente, apreciamos que la objetividad no es el único aspecto al que se debe atender cuando se trata con este léxico, sino también al emisor y al contexto en el que se encuadran. Al hilo de esta apreciación, es de destacar la responsabilidad que conlleva su tratamiento, tanto a nivel ideológico como social, político o cultural. En la presente investigación nos hemos centrado en la figura del actor, el perpetrador del atentado. Es fácil observar la alta cifra de vocablos que emplea la prensa para referirse a este rol, sirviéndose de eufemismos que evitan emplear las "palabras-T" $y$, en este caso en concreto, el vocablo "terrorista" como tal.

Pese a que se puede profundizar aún más en este aspecto, hemos podido evidenciar que esta cautela lingüística seguida por los medios de comunicación cuenta con un gran influjo de las propias organizaciones terroristas (combatientes, militantes, guerreros, etc.). Asimismo, es de destacar el tratamiento de determinadas construcciones y vocablos en contextos caracterizados por su gran carga ideológica. Algunos ejemplos de ello podrían ser "ISIS", "talibán”, "yihad", "ideología takfir" o "ISIL", además de figuras retóricas y metáforas, tales como "construcción del Califato", "enemigos del islam”, "Hijos del Califato” o "cruzada contra el Estado Islámico". Estos ejemplos son parte fundamental de este discurso 
del terror que se hace eco en nuestra sociedad a través de los medios de comunicación. No obstante, muchos de ellos presentan matices léxicos, los cuales en gran medida son consecuencia de cierta influencia cultural, que evidencia un uso y, por tanto, una connotación erróneos de este léxico. Por ello, consideramos determinante el empleo de las siglas, los acrónimos, los neologismos, los arabismos, las palabras de influencia anglosajona $y$, en este estudio en concreto, de los eufemismos, el cual denota la complejidad que supone el tratamiento de este léxico. En cuestiones relacionadas con conflictos de índole terrorista, la transmisión de la información de forma objetiva, así como su correcta traducción a otras lenguas son de vital importancia a nivel político o social, pues destacamos una vez más la carga cultural que conlleva.

Finalmente, pese a la amplia variedad de fuentes bibliográficas que hemos consultado y que han complementado notablemente nuestro estudio, reiteramos, como hemos hecho al principio de esta investigación, la carencia de estudios lingüísticos que reflexionen sobre los dilemas emergentes en el léxico del terrorismo, un concepto cada vez más globalizado para el cual continúa siendo una ardua tarea establecer un consenso a escala internacional en cuanto a su definición.

\section{Referencias bibliográficas}

Álvarez, A. (2019). Terrorismo. Un difícil concepto [Artículo]. Recuperado de https://www.researchgate.net/publication/334520328_Terrorismo_Un_difi cil_concepto.

Casado, M. (1991) Lenguaje y Cultura. Madrid: Síntesis.

Collins Dictionary, Disponible en Web: https://www.collinsdictionary.com/es/.

Crespo, E. (2016). Eufemismo y política: un estudio comparativo del discurso político local británico y español. Pragmalingüística, 24, 8-29.

Diccionario de la Real Academia Española (22 ${ }^{\mathrm{a}}$. Ed). Disponible en Web: http://www.rae.es.

Eco, U. (1986). Golpear el corazón del Estado. La estrategia de la ilusión (150-156). Barcelona: Lúmen.

Flori, J. (2004). Guerra santa, Yihad, cruzada: violencia y religión en el Cristianismo y el Islam. Granada: Universidad de Granada.

Fundéu [en línea]. Disponible en Web: https://www.fundeu.es/.

Gadarian, S. (2010). The politics of threat: How terrorism news shapes foreign policy attitudes. The Journal of Politics, 72(2), 469-483.

Gallud, E. (2005). El eufemismo como instrumento de manipulación social. Revista Comunicación y Hombre, 1, 121-129.

Hoffman, B. (1990). A mano armada. Historia del terrorismo. Madrid: Espasa-Calpe. 
Kalounerová, M. (2015). The Jargon and Terminology of Terrorism. Selected Examples from U.S. Media. Universidad de Bohemia Occidental, Pilsen, República Checa.

Martínez, B. (2001, octubre 17). Eufemismos y disfemismos en los periódicos españoles. Ponencia presentada en Congresos Internacionales de la Lengua Española, Valladolid, España. Recuperado de http://congresosdelalengua.es/valladolid/ponencias/el_espanol_en_la_socied ad/1_la_prensa_en_espanol/martinez_b.htm.

Pegenaute L. (2012). La traducción como herramienta preventiva y paliativa del terrorismo internacional. Lengua, traducción recepción: en honor de Julio César Santoyo, 2, 395-408.

Rodrigo, M. (1991). Los medios de comunicación ante el terrorismo. Barcelona: Icaria.

Shariffan, F. (2007). Politics and/of Translation: Case Studies between Persian and English. Journal of Intercultural Studies, 28(4), 413-424.

Veres, L. (2012). Imagen, terrorismo y argumentación. Revista Iberoamericana de Argumentación, 4, 1-14.

(2006). La retórica del terror. Sobre lenguaje, terrorismo y medios de comunicación. Madrid: Logos.

Wilkinson, P. (1974). Political Terrorism. Londres: Macmillan.

Zurutuza, C. (2006). Información sobre terrorismo: ¿Periodismo o propaganda? Cuadernos de Información, 10, 74-79 
